\title{
A Novel Spatial Image Steganalyzer with Adaptive Channel Attention
}

\author{
Fan Nie*, Chaoyang Zhu \\ Department of Information and Communication, China Electric Power Research Institute, Beijing, China \\ *Corresponding Author.
}

\begin{abstract}
For imagesteganalysis, many studies have showed that the superiority of the convolutional neural network overconventional methods based on artificially designed features. Withthe trend of the fusion of traditional steganalysis methodsand some tricks used in classic computer vision tasks, such asSRNet equipped with residual modules and ZhuNet which usedspatial pyramid pooling, more and more CNN architecturesused for steganalysis are proposed. However, there still are somecharacteristics in most content-adaptive steganographic algorithms such as S-UNIWARD, HUGO, WOW, and tricks in designing network structure whichcan be used for steganalysis. Here, we propose a CNN network framework which can further improve theperformance of spatial imagesteganographic algorithms. First, we utilizemore SRM kernels to initialize the pre-processing layer than previous CNNs, and usean image padding method different from traditional modelsto preserve the integrity of image residuals as much as possible. Next, we use multiple channel attention layers which aim to discriminate the more informational features boosting the detection accuracy of network. Then, we deploy the spatial pyramid poolinglayer before features are fed into the fully-connected layers, aiming to extract more features from the last feature mapsin several scales. Several experiments under different steganographic algorithms show that, the proposed CNN outperforms the other CNN-based steganalyzerssuch as YeNet, XuNet, YedroudjNet,SRNet and ZhuNet.
\end{abstract}

Keywords: Image steganalysis, Convolutional neural network, Channel attention layer

\section{Introduction}

Since the emergence of stegnography and steganalysis, theyhave been developingin competition with each other. Image steganography is away of hiding secretinformation in different domains of an image by slightly making some unnoticeable modification (changing pixel values of image in the spatial domain, and DCT coefficients in the frequency domain).In the past several years, with the advent of multimedias, more and more types of information were used in communication,steganographic algorithms keep developing, from simplest LSB embedding[1,2]to content-adaptive algorithms. Nowadays, those steganographicapproachesequipped with content-adaptive algorithms are more secure, which tend to conceal data in some highly textured regions, aiming to decrease the valuesof the specific pre-defined additional distortion function. Such asWOW[3], S-UNIWARD[4],HUGO[5], and the other methods[6] in spatial domain. In contrast, significant progress has also been made inthe field of image steganalysis, whose propose is to detect the existence of hidden information in images. Before the advent of machine learning techniques, the most powerfulanalyzer of steganographic algorithmswaspixel statistical methods, whichtook advantage of some defects in traditional steganographic algorithms, including RS analysis[7], chiSquare test. Later on, due to the development of machine leanring, several novel steganalysis tools based on the content of images are proposed[8,9].The most famous steganalyzer based on ML classifiers inspatial domain isthe Spatial Rich Model[9]includingits multiple improved variants[10-12], which are regarded as themilestone of the modern steganalysis. Most of these steganalyzersare formed by assembling several submodels constructedby different high-pass filters into a rich bigger model. However, the performace of the conventional steganalyzers with hand-crafted faeturesrelys heavily on the effect of featureengineering, in other word, getting more complete information of image, the model will get better performance. Incontrast, due to limitation of memory and computationalpower, it is impossible to use traditional machine learningmodels directly with a huge amount of

ISSN: 0010-8189

(C) CONVERTER 2020

www.converter-magazine.info 
features which maylead to overfitting or the curse of the dimension.

Benefiting from the development of deep learning, thereare several models of steganalysis using CNN[13-17]. In the traditional computervision tasks, which include image segmentation[18-20],image classification[20,21] and object detection[22], $\mathrm{CNN}$ has been proved that it can efficiently extract thefeatures of images and get better performance, comparedwith conventional methods. Unlike fixed hand-craftedfilters used in traditional steganalysis detectors[9], thesefilters used in different layers of CNN can be optimized automaticallyby back propagation. Therefore, Diverse CNNarchitectures of steganalysis using different ways to improve the detection accuracy are proposed, such as high-pass KV filter[13,14] which is one of the filters in SRM [9], theabsolute value layer(ABS layer)[14], more SRM kernels[15,17], integrating some modules of classical networkarchitectures(such as ResNet[23], InceptionNet[24-26]).

Here, we propose a new network architecture implementedby a convolutional neural network (CNN) named NieNet for steganalysis to capture more comprehensive and discriminative featuresof images. There are multiplenovel characteristics compared with other CNN-basedimage steganalyzers, which are detailed as follows:

(1) In the pre-processing layer, we use 46different filters of SRM(linear and non-linear kernels) toinitialize the kernels to extract the residual of images andmodify the shape of some convolution kernels which are3rd spam kernels mentioned in $[15,17]$. Additionally,before convoluting the input images, the images will bemirrorpadded to impove the robustness of our model andto achieve better accuracy.

(2) We deploy a new network module that combinesessences of two advanced CNN frameworks, ResNetand InceptionNet. This type of network architecture can beused for extracting image features from different perspectives, meanwhile avoid the problem of gradient vanishing.

(3) The success of the attention mechanismin several computer vision tasks demonstrates that attention is important for neural network, we add the channelattention layer to each Res-Inception module similar to[27]. With the help of this layer, the weights of differenttypes of SRM kernels will be assigned dynamically toincrease the stability of our $\mathrm{CNN}$.

On two datasets (BOSSBase[34] and BOWS2[35]), several experiments are conducted, in which we train multiple CNN-based steganalyzers including ours under the same settings and compare their detection performance. And experimental results show our proposed steganalyzer achieves state-of-the-art performance.

\section{Related Works}

Tan [28], uses a convolutional neural network equippedwith four convolutional layers for image steganalysis, plus well-designed initialization using one of the hand-crafted filters. According to characteristicsof Gaussian function, Qian[13] proposed that a CNNarchitecture of steganalysis with Gaussian activation function. But the accuracy of these models is still worsethan traditional models which consist of SRM kernelsand classic machine learning methods (Support VectorMachine, Fisher Linear Discriminator)[9]. Later, $\mathrm{Xu}$ [14]proved that the feasibility of batch normalization layerin $\mathrm{CNN}$-based steganalyzer and introduced absolute value function into the network architecture.

Inspired by previous approaches of image residual extraction, Sedighi[29] proposed a CNN structurewith initialized weights with SRM kernels and well-designed histogramlayers, which projected feature maps into histogrammaps. YeNet[15] proposed a CNN architecture with 30high-pass pre-defined filtersmentioned in SRM[9] for initializing weights of a pre-processing layer plus a truncated activation function (TLU). The Yedrouj-Net[30] achievesbetter performance than YeNet[15] by using multiplemethods of data augmentation. A deep network for image steganalysis equippedwith shortcut connections, SRNet, was proposed in [16] and significantly improve detection accuracy in both spatial domainplus JPEG domain. And it also proved that a model usingrandomly initialized kernels for the first pre-processing layer can also get good performancewith the deeper network structure. Zhu[17] deployed moreadvanced layers including grouped convolution layers andspatial pyramid ISSN: 0010-8189 
pooling layers to a CNN structure, itachieves better accuracy of detection and also regardsimages of arbitrary size as input. Wang[31] combinedmultiple domains of images and proposed WangNet whosethe first layer is initialized with DCT coefficient and moreSRM kernels, their experiments showed that with theincrease of kernels initialized by SRM kernels, the modelcan achieve better accuracy.

\subsection{Architecture}

The architecture of the proposed CNN in demonstrated in Fig 1.It consists of multiple stacked layers including one image pre-processing layer with SRM initilization, several feature extraction layers containing two differenttypes of Res-Inception layers with shortcut connection, a spatial pyramid pooling layermentioned inZhuNet, and there are two fully connected layersgenerating the probability of stego/cover.

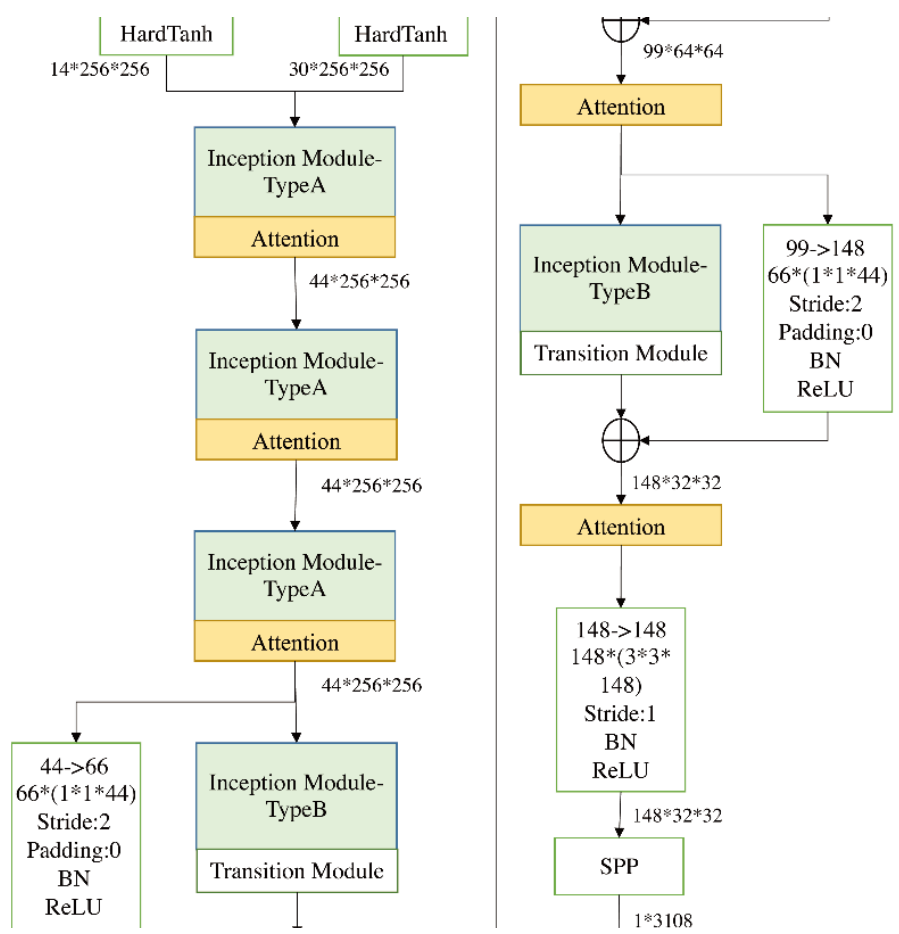

Fig 1: The architecture of our CNN

There are two types of blocks named as 'TypeA Inception' and 'TypeB Inception' shown in Fig.3 to extractspatial correlation with different kernels which have multiple shapes and finally feed these feature maps into fullyconnected layer. Each type of basic blocksconsists of the following steps approximately:

\subsubsection{Convolutional Layer}

We utilizemultiple small convolution kernels (eg. $3^{*} 3$ ) to achieve function of larger kernels instead of directly using large convolutionkernel, preventing too many parameters to slow the time of convergence. By theway, this model can effectively extract different featuremaps by using Inception layer, which contains severalparallel convolution layers with kernels of different size.Specifically, the big differentce between two types ofblocks is that, the maxpooling operation is used in theTypeA block which aims to simulate the function of non-linear kernels mentioned in[9], and the TypeB block is equipped with depth-wise convolution layers similar to [17]aiming to extract features efficiently. And there is a hyperparameter named 'reduction' to be used for controlling thenumber of outputs' channels, according to our setting, thehyper-parameter is set to 2.

\subsubsection{Batch NormalizationLayer}

As $\mathrm{Xu}$ [14] mentions, CNN-based steganalyzer can take advantage of superiority of batch normalization layer, the use of batch normalization allows us to set a larger learning rate, and neural networks can converge quickly

ISSN: 0010-8189 
benefiting from the uniform distribution of features.

\subsubsection{Different Non-Linear Activation Function}

Exceptthe first preprocessing layer followed by a TLU function. For all other blocks in theproposed CNN architecture, we use ReLUas the activation function after Batch Normalization operation, it has been proven in many computer visiontasks to have excellent characteristics, including avoidinggradient vanishing and accelerating model convergence.

\subsubsection{Average Pooling Layer}

In order to decrease the dimension of feature maps, a CNN usually is equipped with several pooling layer for down-sampling feature maps. For most circumstance, it would be max pooling layer, for in the field of CNN-based steganalyzers, average pooling layer is widely chosen for down-sampling features, because of the ability of preserving weakly steganographic signal. Therefore, we choose the average pooling layer for the proposed CNN instead of max pooling layer.

\subsection{Diverse Kernels}

The hiding operation of steganographic algorithms can be regardedas generating designed noises and adding itinto the cover image. As mentionedin YeNet[15], ZhuNet[17], Yedoudj-Net[30], it is a greatidea to use SRM kernels' initialization for steganalysisbased on neural network to extract residual features.However, the common of these former models is that all ofthem use 30 high-pass spam filters of SRM and theirrotated counterparts, we deploy a set of high-pass filters toour CNN's preprocessing layer (30 high-pass filtersof SRM, similarly to YeNet[15], Yedroudj-Net[30] andZhuNet[17], plus 14 non-linear minmax filters). Therefore, our proposed CNN is able to extract more noise residualmaps from input image than other networks, which meansmore comprehensive information can be extracted by our CNN.

\subsubsection{Improved Kernels}

As some existingCNN-based steganalyzersdemonstrates, the filter of size $3 \times 3$, such as "SQUARE $3 \times 3$ ", "EDGE $3 \times 3$ " and the remaining 13 filters of size $5 \times 5$ including "SQUARE5 $\times 5$ ", "EDGE5 $\times 5$ " and "SPAM 3rd" can efficiently extract discriminative features of image. Beside these SPAM kernels, we creatively add some non-linear kernels to the prepro-cessing layer to increase the number of noise residualmaps. In practice, it can be easily implemented by using cross-channel max and min operations. As the additional kernels of SRM[9], we choose the limited numbers of non-linear kernels whichinclude minmax2nd21, minmaxEDGE3 $\times 322 \mathrm{v}$, minmaxEDGE5 $\times 5$ $22 \mathrm{v}$, and minmax3rd 22v. As shown in Fig.2, there are two types of values of different colors performingthe same operations just like linear kernels.
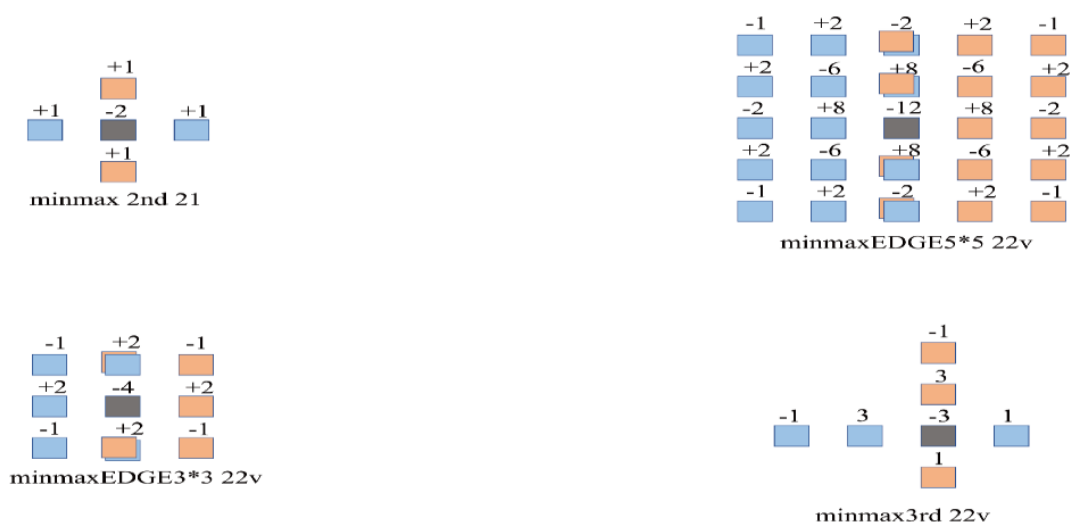

Fig 2: Different non-linear kernels which we choose to use in preprocessing module

\subsubsection{Mirror-PaddingOperation}

Inmanytraditionalcomputer vision models which take the pixel values ofthe image as input, the zero padding

ISSN: 0010-8189

(C) CONVERTER 2020

Www.converter-magazine.info 
operation is mostcommonly used for the sake of simplicity. From theperspective of steganalysis, because of slight differencesbwtween cover images and stego images, modeling image residueswill extract more discriminative featuresusing some hand-crafted filters than directly modelling pixel values. In YedroudjNet[30] andXuNet[14], the weights of convolutional kernels in the preprocessing layerare initialized by some SRM kernels. In ZhuNet[17], there are more SRM filters used for initialization. However,there is adefect among these models, that almost CNN architecturesuse the zero padding operation before convoluting tokeep the size of feature map, but this operation willdemage the quality of our extracted pixels' residues, forinstance, if a zero value is padded to the image, thenthe residue of this image will become large and cannot show the characteristics of this image. Consideringthe characteristic of noise residues extraction and theoperation of some popular steganographic algorithms, suchas HUGO[5], WOW[3], S-UNIWARD[4], which all usethe mirror padding operation to calculate the embeddingcost of the image, doing zero padding operation andoptimizing kernels as usual will damage the quality ofnoise extraction. According to Table 1, by using mirror-padding operation, the performance of CNN will improvea lot.

Table 1 Steganalysis error probability of different optimizing strategies with S-UNIWARD at $0.4 \mathrm{bpp}$

\begin{tabular}{|c|c|c|}
\hline different strategies of optimizing kernels & Our CNN & YeNet \\
\hline mirror_padding optimizing & 0.172 & 0.286 \\
\hline zero_padding optimizing & 0.191 & 0.284 \\
\hline mirror_padding fixed & 0.176 & 0.279 \\
\hline
\end{tabular}

\subsubsection{Inception Module with Separable Convolution}

Inception module has recently been proved that it canimprove the model accuracy significantly in traditionalcomputer vision tasks, such as Inception[24-26],Xception[32]. Because of the wider parallel convolutional layers and shortcuts, thismodule can get more informational features from different scale thangeneral convolution layers but also keep the whole modelfrom getting deeper which will lead gradient vanishing.And in practice, we put these modules into deeper layerswhich can prevent from damage the residual informationof cover/stego, for each Inception module, we just usesmall sized kernels which can efficiently reduce storagespace and accelerate the training process. The another advantage of that is with use of multiple size of filters, the network will benefit from larger receptive field, and discriminate more informational features.
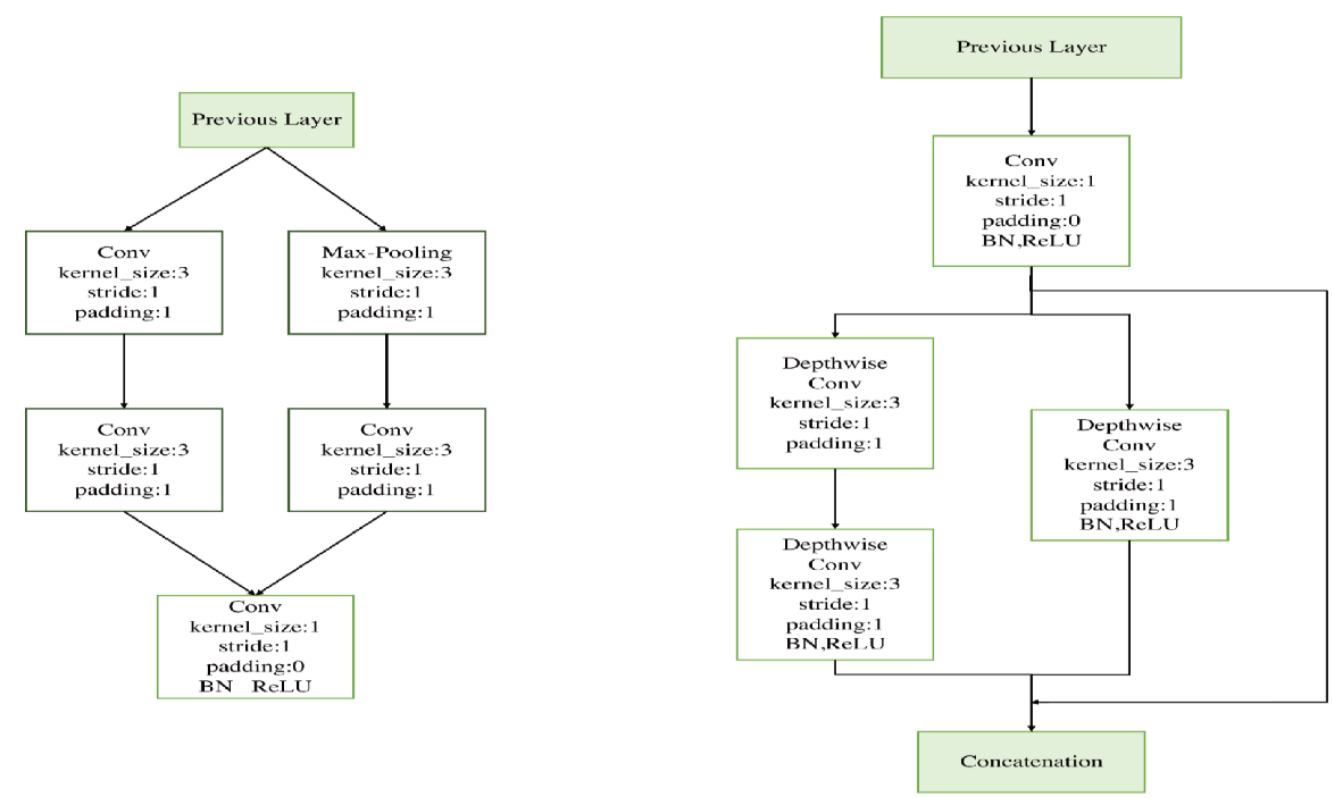

Fig 3: This caption has one line so it is centretwo different Inception modules used to the proposed CNN. Left:TypeA Inception module for shallow layers, Right: typeB Inception module for deeper layers

ISSN: 0010-8189 
As shown in Fig.3, we apply two different types ofInception Module to our proposed network named "IncepTypeA" and "Incep-TypeB", more details are shown inFig.3. In the more shallow layers which are quippedwith 'Incep-TypeA' modules, The use of a combinationof maxpooling operation and convolution operation willnot only extract more features in the larger field, but alsoadd more non-linear features by maxpooling,just like non-linear kernels do in the preprocessing layer. In the deeperlayers which consist of several 'Incep-TypeB' modules, we apply more branches of convolution operations whichinclude grouped convolution operations to these layers,aiming to extract more semantic features efficiently.

\subsubsection{Channel Attention Layer}

Due to the use of differentattention modules which can make the model assign different weights to the content of different regions in the sameinput according to the task like a brain, the performance ofthe model is improved greatly. such as recurrent attentionconvolutional neural network[33], and Residual AttentionNetwork [21]. At the point of steganalysis's view, theactual input to our CNN is several residual maps which arecalculated by the preprocessing layer's multiple kernels.As [9] mentioned, the different residual map processed bydifferent kernels has different performance of steganalysis.Inspired by those ideas, we proposed a new attention layercalled channel attention layer (CAL) used for our CNN-based steganalyzer. The outputs of theCAL the weight for each feature channels which will beused to output weighted feature maps. As shown in Fig.4, Considering the number of additional trainable parameters brought by this layer, weuse a simple strategy to construct this layer similar to[27]which is composed of global average pooling operationand several fully connected layers. But there are somedifferences between the CAL and SE block mentioned in[27]. In the CAL, using a depth-wise convolutional layeradditionally will improve the robustness of this attentionmodule rather than [27].

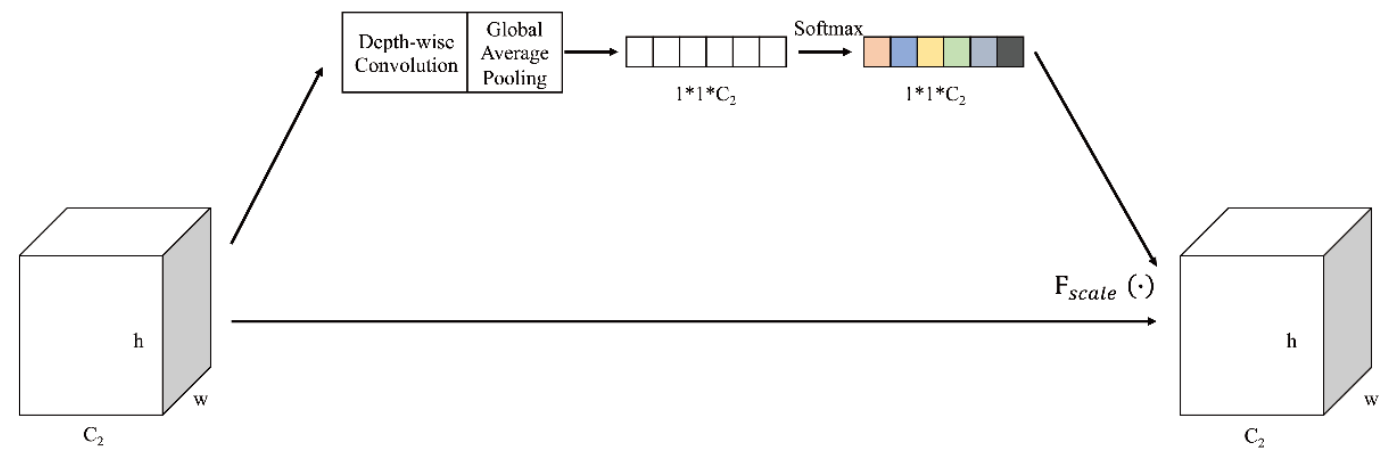

Fig 4: A channel attention module used in the proposed CNN, similar to [27]

To explore the impact of using channel attentionlayers on CNN performance. We designed several CNNstructures for steganalysis mentioned which are appliedproposed attention layers. As shown in Table. 2, due toaddition of the CAL, there is slight improvement amongthese three networks including our proposed CNN. ForZhuNet and Our NieNet under S-UNIWARD at 0.4 bpp,both of them equipped with CAL get better performances.

Table 2 Steganalysis error rate comparison of networks with channel attention layer against multiple steganographic algorithms at $0.4 \mathrm{bpp}$

\begin{tabular}{|l|c|c|}
\hline & S-UNIWARD & WOW \\
\hline
\end{tabular}

ISSN: 0010-8189 


\begin{tabular}{|c|c|c|c|}
\hline \multirow{2}{*}{ SRNet } & with attention & 0.249 & 0.215 \\
\cline { 2 - 4 } & without attention & 0.243 & 0.217 \\
\hline \multirow{2}{*}{ ZhuNet } & with attention & 0.201 & 0.175 \\
\cline { 2 - 4 } & without attention & 0.217 & 0.184 \\
\hline \multirow{2}{*}{ NieNet } & with attention & 0.172 & 0.137 \\
\cline { 2 - 4 } & without attention & 0.186 & 0.152 \\
\hline
\end{tabular}

\subsubsection{Spatial Pyramid Pooling Module}

Spatial pyramidpooling module (SPP) is proposed in Zhu-Net[17] andhelp the model achieve better performance. By using theSPP module, this pooling operation can extract featureinformation in different scales such as $1 \times 1,2$ $\times 2,4 \times 4$,and better model the local features. Besides, Benefiting fromthe fact that spatial pyramid pooling operation can turnthe output of feature maps into fixed sized tensor, our CNN can steganlyze arbitrary-size images.

\subsubsection{The Difference between SRNet, ZhuNet and OurCNN}

The comparison of three CNN-based steganalyzersisillustrated in Table 3. Compared to SRNet, the differences in Our CNN are usinghand-crafted kernels initializing weights of the preprocessing layer proposed in SRM[9], mirror-paddingoperation, deploying two types of Inception Modules (withand without separable convolution) plus spatial pyramidpooling module. Compared to Zhu-Net, the uniquenessof our CNN is using improved kernels and additionalnon-linear kernels for initialization, using mirror-paddingoperation, and using shortcut connection for all left layers.

Table 3 The difference between the SRNet, ZhuNet, and NieNet

\begin{tabular}{|c|c|c|c|c|}
\hline Algorithm & $\begin{array}{l}\text { Preprocessing } \\
\text { module }\end{array}$ & $\begin{array}{c}\text { Padding } \\
\text { operation of } \\
\text { pre-processing } \\
\text { layer } \\
\end{array}$ & $\begin{array}{l}\text { Inception or } \\
\text { shorcut }\end{array}$ & $\begin{array}{l}\text { Pooling before fully } \\
\text { connected layers }\end{array}$ \\
\hline SRNet & $\begin{array}{c}\text { Random } \\
\text { initializationand } \\
\text { update filters }\end{array}$ & zero padding & shortcuts & global average pooling \\
\hline ZhuNet & $\begin{array}{l}\text { initialize with } 30 \\
\text { specific kernels } \\
\text { and update them }\end{array}$ & zero padding & $\begin{array}{l}\text { depthwise } \\
\text { separable } \\
\text { convolutions } \\
\text { and shorcuts }\end{array}$ & spatial pyramid pooling \\
\hline NieNet & $\begin{array}{l}\text { initialize with } 44 \\
\text { specific kernels } \\
\text { and update them }\end{array}$ & mirror padding & $\begin{array}{c}\text { inception } \\
\text { separable } \\
\text { module } \\
\text { and shortcuts }\end{array}$ & spatial pyramid pooling \\
\hline
\end{tabular}

\section{Experiments}

Several experiments are conductedto show the effectiveness of our proposedCNN. We compare our model with several CNN-based steganalyzers: XuNet[14], YeNet[15], YedroudjNet[30],SRNet[16], ZhuNet[17]. All networks are trained andtested on the same datasets and same steganographic algorithms (WOW[3], S-UNIWARD[4]) for fair comparison.

\subsection{The Environments}

For steganographic algorithms, all of them are implemented on the publicly available codes. We choose the onesin Matlab implementation with random embedding key.As [15] mentioned, if the embedding key is unchanged,the generalization of networks will decrease dramatically.And by using Nvidia Tesla V100 graphic card with 32GB memory for training, we can use larger batch size oftraining images to get more stable parameters of batchnormalization layer.

\subsection{Datasets}

ISSN: 0010-8189 
In this paper, we use the combination of two standarddatasets which are commonly used for steganalysis to trainthese networks and test the performance of these networks. The first source of images is from the BOSSBase[34], it contains10,000 512×512 grayscaleimages taken by several devicesand is commonly used in steganalysis. The other is BOWS2[35], it contains 10,000 $512 \times 512$ grayscale imageswhose distribution is similar to BOSSBase. Based onconsideration of GPU memory and batch size of trainingprocess, we decide to conduct experiments on resizedimages of $256 \times 256$ pixels created by Matlab with default settings. The setting of datasetsof training and testing will be detailed later.

\subsection{Experimental Parameters Setting}

Stochastic gradient descent (SGD) approach is widely used in several field of deep learning as the optimizing algorithm. Therefore, at the training phase of our image steganalyzer, we choose SGD to optimize our model. According to previous experiences and memory limitation of our graphic processing unit, batch size of our training data is going to be set to 32 (16 cover/stego image pairs). The ultimate goal of our training phase is to minimize the cross-entropy loss of outputs of our CNN.

On our dataset, the model will be trained for 250 epochswith a learning rate of $r=0.0001$. The learningrate will be modified (divided by 5) at epoch 100,150,200respectively. According to our practical experience, theCNN training will converge totally at about 200 epochs. We train and test all models used in this paper for several times using same datasetsetting (uniform training/validation/testing sets). Thefinal experimental results are concluded by averaging thesetesting results. The metric of these CNN models was defined by thesteganalysis error rates $P_{e}=1$ $\mathrm{P}_{\mathrm{acc}}$.

\subsection{Experimental Results}

\subsubsection{BOSSBase Only}

As shown in Table4, the performance of several steganalyzers are reported in our uniform experiment settings. First, cover images of BOSSBase are randomly divided into three parts: 4000 cover/stego image pairs are used for training networks, 1000 cover/stego image pairs are used as validation set and 5000 pairs as test set. In this table, we use two steganographic algorithms S-UNIWARD and WOW to conduct experiments under different payloads.

Table 4 Steganalysis error rate comparison.

All networks are trained and tested on BOSSBase with same settings

\begin{tabular}{|c|c|c|c|c|}
\hline Algorithm & \multicolumn{2}{|c|}{ WOW } & \multicolumn{2}{c|}{ S-UNIWARD } \\
\hline Payload/bpp & 0.2 & 0.4 & 0.2 & 0.4 \\
\hline XuNet & 0.331 & 0.283 & 0.354 & 0.312 \\
\hline YeNet & 0.324 & 0.277 & 0.341 & 0.298 \\
\hline YedroudjNet & 0.317 & 0.252 & 0.327 & 0.279 \\
\hline SRNet & 0.251 & 0.204 & 0.294 & 0.243 \\
\hline ZhuNet & 0.215 & 0.176 & 0.258 & 0.217 \\
\hline NieNet & 0.223 & 0.167 & 0.247 & 0.213 \\
\hline
\end{tabular}

In different experiment settings including different algorithms and different payloads, our CNN has gotten better detection performance over the other CNN-based steganalyzer. Specifically, under the circumstance of same payload and different algorithms.(S-UNIWARD and WOW), the detection performance of our proposed CNN is ahead of other steganalyzers except ZhuNet, the proposed CNN and ZhuNet get comparable detection performance under same payload.(such as $21.5 \%$ versus $22.3 \%$ under 0.2 bpp with WOW, $17.6 \%$ versus $16.7 \%$ under 0.4 bpp with S-UNIWARD)Similar to other networks under same algorithm and different payloads, our proposed CNN can get better performance under larger payload.(for example $21.3 \%$ versus $24.7 \%$ under S_UNIWARD with 0.2 bpp and $0.4 \mathrm{bpp}$ )We speculatethat the reason for this phenomenon is that because of a small number of training datasets, these attention modules used are not well trained, when the number of training samples goes larger, this

ISSN: 0010-8189 
phenomenon will not exist, and the next part of the experiment also verifies this idea.

\subsubsection{BOSSBase and BOWS2}

For well training networks with larger number of training samples, we add extra image sources BOWS2 (containing 10000 images which has the similar distribution with BOSSBase) into our training set. Therefore, the training set now is composed of 14000 pairs of cover/stego images.

Table 5Steganalysis error rate comparison.

All networks are trained and tested on BOSSBase + BOWS2 with same settings

\begin{tabular}{|c|c|c|c|c|}
\hline Algorithm & \multicolumn{2}{|c|}{ WOW } & \multicolumn{2}{c|}{ S-UNIWARD } \\
\hline Payload/bpp & 0.2 & 0.4 & 0.2 & 0.4 \\
\hline XuNet & 0.318 & 0.243 & 0.346 & 0.234 \\
\hline YeNet & 0.284 & 0.211 & 0.367 & 0.247 \\
\hline YedroudjNet & 0.278 & 0.177 & 0.361 & 0.229 \\
\hline SRNet & 0.256 & 0.156 & 0.348 & 0.218 \\
\hline ZhuNet & 0.241 & 0.127 & 0.286 & 0.164 \\
\hline NieNet & 0.237 & 0.131 & 0.261 & 0.152 \\
\hline
\end{tabular}

As shown in Table 5, under the circumstance of larger training set, the performance of all networks has been improved simultaneously, in almost settings, our proposed CNN has outperformed other CNN-based steganalyzers. As we say above, once our attention modules can be well trained with larger datasets, our CNN will get better detection performance.

\section{Conclusion}

In this article, we apply more techniques used for traditional computer vision tasks to architecture of CNN-based steganalysis. The advantages of our proposed CNNare following: First, wedeploy more pre-defined convolution kernels and use a novel paddingoperation in preprocessing layer, these operations make our CNN extract more discriminative features. More convolution kernels enhance the robustnessof this model. Second,we continuously combine separableconvolution mentioned in [17] and Inception Module toextract channel correlation. Finally we use channel attentionlayer which will increase the computation slightly to makeour model allocate different channel different weight. Byusing these tricks, the network performance is improved.Additionally, using SPPmodule, an arbitrary sized imagecan be steganalyzed by the CNN. In the furture, the application of attention mechanism on image steganalysis should be exploited thoroughly, and we will design more powful attention module for image steganalysis.

\section{Acknowledgements}

This research was partly supported by the National Key Research and Development Program of China (Grant No. 2020YFB0905900).

\section{References}

[1] H.-C. Wu, N.-I. Wu, C.-S. Tsai, and M.-S. Hwang, "Image steganographic scheme based on pixelvalue differencing and lsb replacement methods," IEE Proceedings-Vision, Image and Signal Processing, vol. 152, no. 5, pp. 611-615, 2005.

[2] Y.-K. Lee and L.-H. Chen, "High capacity image steganographic model," IEE Proceedings-Vision, Image and Signal Processing, vol. 147, no. 3, pp. 288-294, 2000.

[3] V. Holub and J. Fridrich, "Designing steganographic distortion using directional filters," in 2012 IEEE International workshop on information forensics and security (WIFS). IEEE, 2012, pp.234-239.

[4] V. Holub, J. Fridrich, and T. Denemark, "Universal distortion function for steganography in an arbitrary domain,” EURASIP Journal on Information Security, vol. 2014, no. 1, p. 1, 2014.

ISSN: 0010-8189 
[5] T. Pevnỳ, T. Filler, and P. Bas, "Using high-dimensional image models to perform highly undetectable steganography,” in International Workshop on Information Hiding. Springer, 2010, pp. 161-177.

[6] B. Li, S. Tan, M. Wang, and J. Huang, "Investigation on cost assignment in spatial image steganography," IEEE Transactions on Information Forensics and Security, vol. 9, no. 8, pp. 1264$1277,2014$.

[7] A. D. Ker, "Quantitative evaluation of pairs and rssteganalysis," in Security, Steganography, and Watermarking of Multimedia Contents VI, vol. 5306. International Society for Optics and Photonics, 2004, pp. 83-97.

[8] T. Pevny, P. Bas, and J. Fridrich, "Steganalysis by subtractive pixel adjacency matrix," IEEE Transactions on information Forensics and Security, vol. 5, no. 2, pp. 215-224, 2010.

[9] J. Fridrich and J. Kodovsky, "Rich models for steganalysis of digital images," IEEE Transactions on Information Forensics and Security, vol. 7, no. 3, pp. 868-882, 2012.

[10] V. Holub, J. Fridrich, and T. Denemark, "Random projections of residuals as an alternative to cooccurrences in steganalysis," in Media Watermarking, Security, and Forensics 2013, vol. 8665. International Society for Optics and Photonics, 2013, p. 86650L.

[11] T. Denemark, V. Sedighi, V. Holub, R. Cogranne, and J. Fridrich, "Selection-channel-aware rich model for steganalysis of digital images," in 2014 IEEE International Workshop on Information Forensics and Security (WIFS). IEEE, 2014, pp. 48-53.

[12] M. Goljan, J. Fridrich, and R. Cogranne, "Rich model for steganalysis of color images," in 2014 IEEE International Workshop on Information Forensics and Security (WIFS). IEEE, 2014, pp. 185-190.

[13] Y. Qian, J. Dong, W. Wang, and T. Tan, "Deep learning for steganalysis via convolutional neural networks," in Media Watermarking, Security, and Forensics 2015, vol. 9409. International Society for Optics and Photonics, 2015, p. 94090J.

[14] G. Xu, H.-Z. Wu, and Y.-Q. Shi, "Structural design of convolutional neural networks for steganalysis," IEEE Signal Processing Letters, vol. 23, no. 5, pp. 708-712, 2016.

[15] J. Ye, J. Ni, and Y. Yi, "Deep learning hierarchical representations for image steganalysis," IEEE Transactions on Information Forensics and Security, vol. 12, no. 11, pp. 2545-2557, 2017.

[16] M. Boroumand, M. Chen, and J. Fridrich, "Deep residual network for steganalysis of digital images," IEEE Transactions on Information Forensics and Security, vol. 14, no. 5, pp. 1181-1193, 2018.

[17] R. Zhang, F. Zhu, J. Liu, and G. Liu, "Depth-wise separable convolutions and multi-level pooling for an efficient spatial cnn-based steganalysis," IEEE Transactions on Information Forensics and Security, vol. 15, pp. 1138-1150, 2019.

[18] O. Ronneberger, P. Fischer, and T. Brox, "U-net: Convolutional networks for biomedical image segmentation," in International Conference on Medical image computing and computer-assisted intervention. Springer, 2015, pp. 234-241.

[19] V. Badrinarayanan, A. Kendall, and R. Cipolla, "Segnet: A deep convolutional encoder-decoder architecture for image segmentation," IEEE transactions on pattern analysis and machine intelligence, vol. 39, no. 12, pp. 2481-2495, 2017.

[20] D. Ciregan, U. Meier, and J. Schmidhuber, "Multi-column deep neural networks for image classification," in 2012 IEEE conference on computer vision and pattern recognition. IEEE, 2012, pp. 3642-3649.

[21] F. Wang, M. Jiang, C. Qian, S. Yang, C. Li, H. Zhang, X. Wang, and X. Tang, "Residual attention network for image classification," in Proceedings of the IEEE conference on computer vision and pattern recognition, 2017, pp. 3156-3164.

[22] P. Viola, M. Jones et al., "Robust real-time object detection," International journal of computer vision, vol. 4, no. 34-47, p. 4, 2001.

[23] K. He, X. Zhang, S. Ren, and J. Sun, "Deep residual learning for image recognition," in Proceedings of the IEEE conference on computer vision and pattern recognition, 2016, pp. 770-778.

[24] C. Szegedy, W. Liu, Y. Jia, P. Sermanet, S. Reed, D. Anguelov, D. Erhan, V. Vanhoucke, and A. Rabinovich, "Going deeper with convolutions," in Proceedings of the IEEE conference on computer vision and pattern recognition, 2015, pp. 1-9.

ISSN: 0010-8189 
[25] S. Ioffe and C. Szegedy, "Batch normalization: Accelerating deep network training by reducing internal covariate shift," arXiv preprint arXiv:1502.03167, 2015.

[26] C. Szegedy, V. Vanhoucke, S. Ioffe, J. Shlens, and Z. Wojna, "Rethinking the inception architecture for computer vision," in Proceedings of the IEEE conference on computer vision and pattern recognition, 2016, pp. 2818-2826.

[27] J. Hu, L. Shen, and G. Sun, "Squeeze-and-excitation networks," in Proceedings of the IEEE conference on computer vision and pattern recognition, 2018, pp. 7132-7141.

[28] S. Tan and B. Li, "Stacked convolutional auto-encoders for steganalysis of digital images," in Signal and Information Processing Association Annual Summit and Conference (APSIPA),2014 Asia-Pacific. IEEE, 2014, pp. 1-4.

[29] V. Sedighi and J. Fridrich, "Histogram layer, moving convolutional neural networks towards featurebased steganalysis,” Electronic Imaging, vol. 2017, no. 7, pp. 50-55, 2017.

[30] M. Yedroudj, F. Comby, and M. Chaumont, "Yedroudj-net: An efficient cnn for spatial steganalysis," in 2018 IEEE International Conference on Acoustics, Speech and Signal Processing (ICASSP). IEEE, 2018, pp. 2092-2096.

[31] Z. Wang, M. Chen, Y. Yang, M. Lei, and Z. Dong, "Joint multi-domain feature learning for image steganalysis based on cnn," EURASIP Journal on Image and Video Processing, vol. 2020, no. 1, pp. 112, 2020.

[32] F. Chollet, "Xception: Deep learning with depthwise separable convolutions," in Proceedings of the IEEE conference on computer vision and pattern recognition, 2017, pp. 1251-1258.

[33] J. Fu, H. Zheng, and T. Mei, "Look closer to see better: Recurrent attention convolutional neural network for fine-grained image recognition," in Proceedings of the IEEE conference on computer vision and pattern recognition, 2017, pp. 4438-4446.

[34] P. Bas, T. Filler, and T. Pevnỳ, "” break our steganographic system": the ins and outs of organizing boss," in International workshop on information hiding. Springer, 2011, pp. 59-70.

[35] P. Bas and T. Furon, "Bows-2," 2007. 